Reprod. Nutr. Dévelop., 1987, 27 (1 B), 303-304.

\title{
Influence relative de quelques facteurs zootechniques sur les paramètres sanguins autour du vêlage chez les vaches laitières
}

\author{
F. ANDRE, S. BAZIN (*)
}

Laboratoire de Méthodologie diagnostique, E.N.V., B.P. 527, 44026 Nantes Cedex. (*) I.T.E.B., 149, rue de Bercy, 75595 Paris Cedex 12.

Summary. In order to define the practicability of metabolic profiles as feeding controls, some blood parameters were studied by multivariate analysis around calving time. The results showed that physiological stage was the most frequent criterion of the blood parameter value; cow, lactation range, maximal production, weight loss after calving and feeding interacted also.

L'appréciation de l'alimentation à travers les profils métaboliques pose le problème de la méconnaissance de tous les facteurs intervenant dans la valeur d'un paramètre sanguin. Ce travail a pour but de montrer qu'outre le stade physiologique, d'autres facteurs zootechniques ont un effet sur certains paramètres sanguins.

Matériel et méthodes. Quatre lots de $(25 \pm 2)$ vaches (production moyenne $>25 \mathrm{~kg} / \mathrm{v} / \mathrm{j}$ ) ont été utilisés pour comparer divers types de rations en période de tarissement et lactation : le lot 1 recevait une ration complète (ensilage de mais et concentré) ; le lot 2 de l'ensilage de maïs et du complément de distribution automatique ; le lot 3 était nourri à l'herbe pendant le tarissement, une ration complète (ensilage de maïs et concentré) pendant la lactation; le lot 4 recevait de l'ensilage de maïs additionné de concentré pendant la lactation. L'apport de concentré était calculé sur la production maximale de la lactation précédente et ajusté à la production réelle à la troisième semaine. Les essais ont été effectués dans deux stations expérimentales : Trinottières pour les lots 1 et 2, Crécom pour les lots 3 et 4 . Quatre prélèvements sanguins à la veine jugulaire ont été effectués (jours $-30,+10,+30,+45$ par rapport au velage), $2 \mathrm{~h}$ après le repas du matin. Les plasmas ont été séparés et congelés dans I'heure suivant le prélèvement après centrifugation des prélèvements $(10 \mathrm{~min} ; 3000 \mathrm{~g})$. Pour le dosage des corps cétoniques, un prélèvement de sang $(5 \mathrm{ml})$ sur $5 \mathrm{ml}$ d'acide perchlorique $(1 \mathrm{~N})$ a été réalisé et congelé immédiatement. Treize paramètres sanguins ont été étudiés par méthodes enzymatiques: glucose, $\beta$-hydroxybutyrate, acétoacétate, lactate, acides gras non estérifiés, cholestérol total et urée; ou colorimétriques : phospholipides, protéines totales, albumine, créatinine, calcium et magnésium. Ont été également relevés : l'âge des animaux, leur production maximale et la perte moyenne de poids après vêlage (pesée des animaux à 1 et 56 jours).

Résultats et discussion. L'analyse de variance toutes valeurs confondues met en évidence (tabl. 1) un effet significatif majeur du stade physiologique pour une hypoglycémie et une hypercétonémie associées à une lipomobilisation mise en évidence par l'augmentation du taux d'acides gras non estérifiés. Cette augmentation ne semble pas persister après le premier mois ; en revanche, pendant le second mois de lactation, les taux de cholestérol et phospholipides augmentent. La complémentation protéique semble entraîner une élévation progressive des taux d'urée et de protéines totales. L'analyse de variance sur les résultats à chaque stade physiologique dans chaque ferme montre qu'à certains stades des effets significatifs de l'åge, de la production maximale, de la perte de poids après le vêlage aussi bien que de la ration sont observés (tabl. 2). Les différences de régi- 
mes au tarissement ( $C R, \mathrm{j}-30)$ modifient la valeur des paramètres énergétiques et de l'urée mais cette action se manifeste encore au début de lactation alors que les rations sont identiques $(C R, j+30)$. Une différence dans le mode de distribution du concentré à niveau égal semble influer sur les taux de protéines totales et urée (TR, $+10,+30 \mathrm{j}$ ). Enfin, si la perte de poids après vêlage et la production maximale ont un effet sur la valeur de paramètres du métabolisme énergétique ( $\beta$-hydroxybutyrate, acides gras non estérifiés) ou azotés (protéines totales, urée), un effet de l'âge des animaux sur ces mêmes paramètres peut parfois être observé.

TABL. 1. - Valeurs moyennes des paramètres sanguins pour lesquels un effet significatifs $(P<0,05)$ du stade physiologique peut être mis en évidence par analyse de variance.

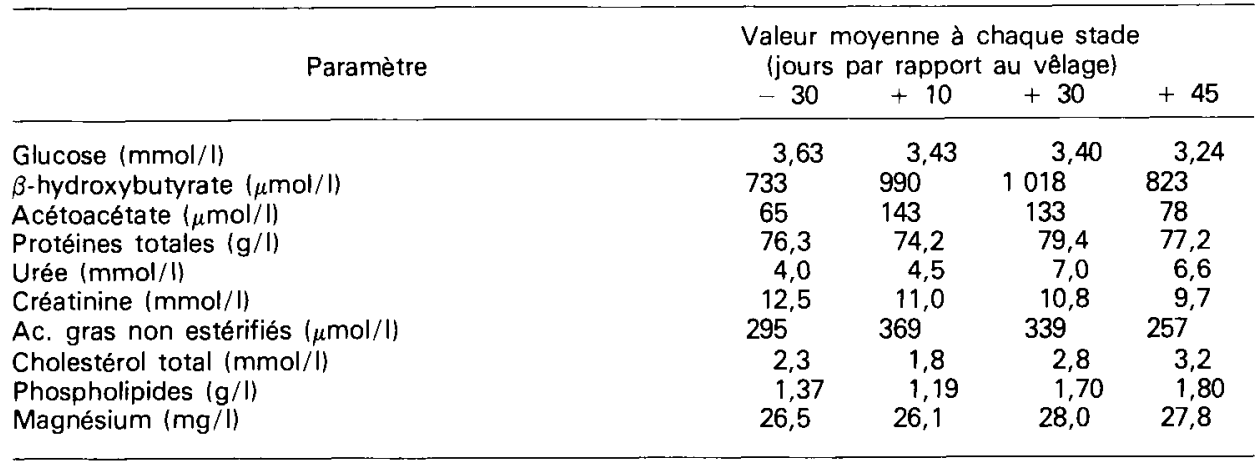

TABL. 2. - Analyse à 4 stades physiologiques des effets significatifs $(\mathrm{P}<0,05)$ de quelques caractéristiques des animaux et du type de ration sur les paramètres sanguins.

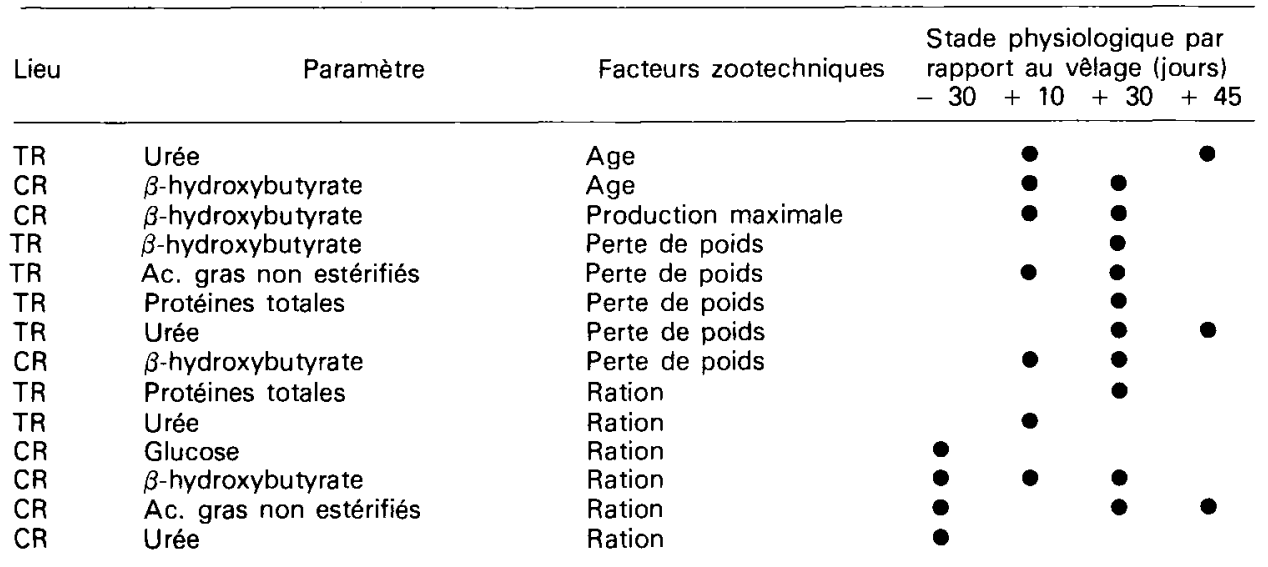

présence d'un effet significatif du facteur concerné sur le paramètre par analyse de variance au stade donné.

Conclusion. La valeur des paramètres sanguins chez les vaches laitières autour du vêlage dépend de la ration et de nombreux autres facteurs : le stade physiologique, le niveau de production, la perte de poids après le vêlage et l'âge des animaux, dont il devra être tenu compte lors de leur interprétation.

L'utilisation des profils métaboliques en alimentation animale. CAAA, Ed. Adeprina, Paris, 1980. The use of blood metabolites in animal production. B.S.A.P., Milton Keynes, 1978. 